\title{
A case study of Phela an African herbal medicine on evidence based research
}

Makhotso Lekhooa' ${ }^{1}$, Andrew Walubo ${ }^{2}$, Gilbert Matsabisa ${ }^{2}$

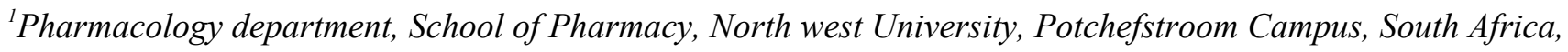 \\ ${ }^{2}$ Pharmacology department, University of the Free State, Bloemfontein, South Africa
}

BACKGROUND: Phela is an African herbal medicine derived from four South-African plants. For generations, combination of these plants has been used successfully to alleviate symptoms in patients with a wasting syndrome, commonly seen in HIV/AIDS patients. Furthermore, observational studies indicated that Phela could have an effect on the immune system. Hence, the aim of this study was to evaluate the safety and clinical efficacy of Phela through evidence based research.

METHODS: Chromatographic methods were developed for the quality control of Phela. Thereafter, evaluating the in vivo immunomodulatory properties of Phela and the mechanism of action was studied in an immune-suppressed rat model. The effect of treatment with Phela at a dose of $15.4 \mathrm{mg} / \mathrm{kg} /$ day for 21 days on the induced immune suppression of rats with either cyclosporine-A, cyclophosphamide and dexamethasone was investigated. The immunological markers assessed were IL-2, IL-10, IgG, IgM, CD4, CD8 counts and clinical parameters.

RESULTS: Three bands with retention factors of $0.24,0.31$, and 0.92 respectively were visible on the thin layer chromatogram. High performance liquid chromatography with a diode array detector separated two marker peaks (at 9.35 and 24.5 minutes), whereas four marker peaks (at 12.2, 22.3, 32.9 and 38.3 minutes) were exhibited with the fluorescence detector. Phela reversed cyclophosphamide suppression by increasing the IL-10, IgG and IgM markers, whereas IL-2, IL -10, CD4 and CD8 counts were increase in cyclosporine-induced suppression. However, no effect was observed for the dexamethasone treated rats. These results suggests a mechanism of action that involves both cell and humoral mediated mechanisms. Modulation of cell mediated immunity by Phela, presents the potential of value in diseases such as HIV and $\mathrm{TB}$, where the cell mediated immunity is compromised.

CONCLUSION: The results of the current study indicate promising modulation of cell mediated immunity by Phela and the potential to be used as an immune booster for HIV positive patients. This study supports the importance of evidenced based pharmacological research as a tool to validate the safety, efficacy and clinical relevance of natural products that have been used from one generation to another for their medicinal value. 